\title{
All-PM Fiber Loop Mirror Interferometer Analysis and Simultaneous Measurement of Temperature and Mechanical Vibration
}

\author{
Daniel Leandro and Manuel Lopez-Amo, Senior Member, IEEE.
}

\begin{abstract}
In this work, a new all-polarization maintaining (PM) fiber loop mirror interferometer is proposed and validated as temperature and mechanical vibration sensor. The scheme employs the arms of a PM coupler as communication fibers, fused with a relative angle of $45^{\circ}$ to the sensing fiber. The length of the arms is equal so their contribution in canceled, obtaining a total transfer function exclusively defined by the sensing fiber. The capabilities of the system as sensor are tested, achieving mechanical vibration and temperature sensing without crosstalk between measurands. In this manner, vibration frequencies up to $1.5 \mathrm{kHz}$ have been monitored using a commercial interrogator with a scan rate of $1 \mathrm{~Hz}$ and a technique based on the fast Fourier transform. Additionally, the immunity of the setup to external perturbations in the communication fibers is studied and compared to the conventional approach.
\end{abstract}

Index Terms - Fiber loop mirror (FLM), multiplexing, optical fiber sensors, photonic crystal fiber (PCF), Sagnac interferometer.

\section{INTRODUCTION}

High-birefringence fiber loop mirrors (HiBi FLMs), sometimes referred in literature as Sagnac interferometers (even if they are not used to detect rotation), are a fiber optic device that has been effectively used in many applications such as optical filtering for fiber lasers or communications [1]. The phase difference of the light counter-propagating along the loop in a HiBi-FLM depends on the birefringence of the $\mathrm{HiBi}$ fiber. As a consequence, externally induced variations on the birefringence can be detected by analyzing the interference, allowing the use of the system as sensor. Among other parameters, temperature and axial-strain variations can be directly measured [2]. Moreover, multi-parameter sensing can be achieved by using special fibers as photonic crystal fiber with two asymmetric cores [3] or small core multi-

Manuscript received 31 July, 2017. This work has been supported by the Spanish Comisión Interministerial de Ciencia y Tecnología within projects TEC2013-47264-C2-2-R and TEC2016-76021-C2-1-R and by the Institute of Smart Cities by means of a postdoctoral fellowship.

D. Leandro and M. Lopez-Amo are with Public University of Navarra: Institute of Smart cities and Department of Electric and Electronic Engineering, Campus Arrosadia S/N, 31006, Navarra, Spain. (e-mail: daniel.leandro@unavarra.es).

Copyright (c) 2017 IEEE. Personal use of this material is permitted. However, permission to use this material for any other purposes must be obtained from the IEEE by sending a request to pubs-permissions@ieee.org. structured fiber [4].

One of the most extended techniques to interrogate $\mathrm{HiBi}$ FLMs (and other interferometric sensors) is just by monitoring the spectral shift of a fringe of the interference. However, when multiple interference patterns are combined in a single response (e.g. in multiplexing schemes or multi-interference interferometers), this approach generally become cumbersome or unreliable. In that case, a method based on the fast Fourier transform (FFT) can be employed to identify each particular component in the spatial-frequency domain that contributes to the interference formation [5]. The FFT interrogation-based technique has been used to multiplex in a single network different interferometric sensors, such as cascaded chirped long period gratings [6], microfiber knot resonators [7], inline photonic crystal fiber (PCF) sensors [8], Fizeau strain sensors [9], graded-index multimode fiber Fabry-Pérot cavities [10], HiBi FLMs [11] and also multiple HiBi sensing fibers in a compound fiber loop mirror [11-13]. Additionally, this method provides more than 100 times greater resolution by monitoring the phase of the FFT instead of the spectral shift of a single fringe in the optical spectrum (the conventional solution) [13].

In general terms, a HiBi FLM offers important advantages over other fiber optic sensors such as high sensitivity or dependence of the interference period only on the length of the $\mathrm{HiBi}$ fiber. In addition, the ability of using special fibers as sensing elements significantly enhances their sensing capacities. Nevertheless, HiBi FLMs suffer from two remarkable constraints over other fiber optic sensor devices like fiber Bragg gratings. Firstly, the maximum number of HiBi FLM sensors that can be multiplexed in a single network is reduced. Up to date, only a few investigations have addressed this aspect [11-15]. The second practical limitation is related with the use of single-mode fiber (SMF) to interconnect the HiBi fiber in the loop; since a SMF optical coupler is typically employed in the conventional version of the HiBi FLM. Consequently, a polarization controller (PC) is required to set the angle difference of the light at the ports of the optical coupler. Furthermore, this effect might become particularly bothersome in multiplexing networks, because one PC per sensing element has to be adjusted [11,13].

This constraint can be avoided by forming HiBi FLM interferometers using exclusively polarization-maintaining (PM) fiber. The first all-PM FLM scheme was proposed as temperature sensor in [16] but more complex all-PM 
multiplexing setups can also be formed by an appropriate design: considering the fiber lengths and the rotation angles between the fiber splices $[12,13]$. These schemes do not need any polarization control inside the loop. That entails that no adjustment of the sensor is required to operate, which together with the enhanced multiplexing capacity significantly increases its practical applicability.

Moreover, the interrogation of all-PM FLMs can be achieved by a commercial FBG interrogator adapted by software to employ the FFT-based interrogation technique [11]. This simplifies the interrogation of multiplexing setups and due to the FFT analysis, it also offers a high resolution [13]. On the other hand, all-PM FLMs require PM optical couplers, which unbalanced arms generate its own interference contribution, becoming sensing elements themselves $[12,13,16]$. That might not always be desirable: e.g. if specific fibers have to be used as sensing elements. In that case, the undesired contribution can be discarded, but it increases in a factor of two in the interference contributions, halving the maximum number of sensing elements that can be multiplexed without crosstalk.

In this work, this influence is avoided by using an all-PM balanced setup in which the fiber arms of the PM coupler compensate between them, without generating any interference contribution and allowing a more efficient use of the spatial-frequency spectrum. An extra fiber section is included as sensing element, obtaining as a result the equivalent version of the conventional HiBi FLM without the need of a polarization controller. Preliminary results presented in [17] have been completed and extended. Initially, the setup is theoretically analyzed; matching with the simulations performed using the three-section analysis. After that, simultaneous temperature and vibration measurements have been achieved without crosstalk, using the FFT-based interrogation technique. Finally, a comparison of the behavior of a conventional HiBi FLM and the proposed all-PM FLM is performed, showing superior immunity to external perturbations in the communication fibers.

\section{THEORETICAL CONSIDERATIONS AND PRINCIPLE OF OPERATION}

\section{A. Introduction to conventional HiBi fiber loop mirrors}

In order to assemble a conventional one-section highbirefringence fiber loop mirror (HiBi FLM), it is only required an optical fiber coupler, which output ports are connected in a loop by a section of high-birefringence fiber and a polarization controller, as displayed in Fig. 1 [1]. The light injected in the coupler exits through the output ports and counter-propagates along the loop. Before reaching the $\mathrm{HiBi}$ fiber, a polarization angle mismatch is induced at one end of the HiBi fiber section by a polarization controller. Due to the birefringence of the fiber, light travelling clockwise and anticlockwise suffer a phase shift, which results in the generation of an interference pattern when recombined at the optical coupler.

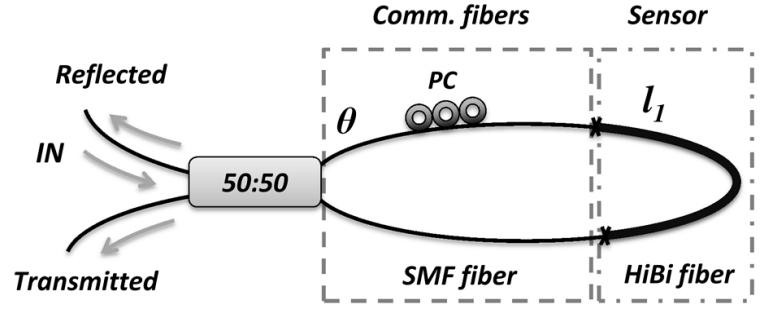

Fig. 1. Schematic of a conventional one-section HiBi FLM.

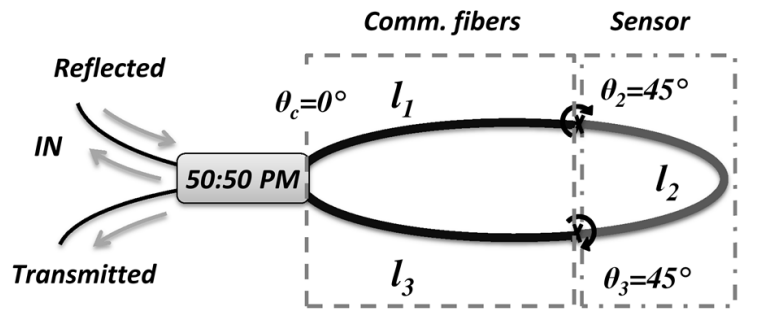

Fig. 2. Schematic of the proposed all-PM FLM.

In this manner, the transfer function $T$ (and the complementary reflection $R$ ) of a simple conventional $\mathrm{HiBi}$ FLM can be described as follows:

$$
T=1-R=\left[\sin \theta \cos \left(\beta_{1} / 2\right)\right]^{2}
$$

Where:

$$
\beta_{n}=\frac{2 \pi \cdot l_{n} \cdot b_{n}(\lambda)}{\lambda}+\Delta \phi_{n}
$$

And $\theta$ is the angle difference of the polarization states at the ports of the coupler (set by the polarization controller), $l_{n}$ is the length of the HiBi fiber, $\lambda$ denotes the wavelength of operation and $b_{n}(\lambda)$ is the birefringence of the fiber. This birefringence is slightly dependent on the wavelength; however, in this work it will be considered constant in the range of operation around a central wavelength $\lambda_{0}$. Moreover, instead of birefringence, it will be employed in the simulations the beat-length of the HiBi fiber, $L_{B}$ that is related with the birefringence as follows: $b(\lambda)=\lambda_{0} / L_{B}$. Finally, $\Delta \phi_{n}$ represents the phase shift induced by external perturbations in the $n$-th $\mathrm{HiBi}$ fiber section (such as temperature or strain changes) [2]. In this manner, the obtained interference presents a sinusoidal pattern with a period given by the relationship between birefringence and the fiber length. The amplitude of the sinusoidal is set by the polarization controller.

This conventional HiBi fiber loop mirror has been proposed for sensing and filtering applications in numerous works up to date. Nevertheless, one important constraint, not usually mentioned in literature, is that polarization states of the light that propagates along single-mode fiber vary gradually in a random and uncontrolled manner depending on external factors such as temperature or bending. In practice, this implies that the single-mode fiber of the loop must remain steady after the polarization controller is adjusted. Even in that case, the polarization controller should be adjusted from time to time, which is not desirable for long-term measurements. 


\section{B. Equivalent all-PM fiber loop mirror}

To avoid the weaknesses related with the use of singlemode fiber in the scheme, an all-PM setup can be employed. A possible approach to obtain an all-PM FLM with a simple transfer function is to use an unbalanced setup where the arms of a PM optical coupler are fused between them with a $90^{\circ}$ angle [16]. However, this solution can considerably limit the sensing performance of the system. Firstly, the sensing fiber type is forced to be the one given by the PM optical coupler, which might be a serious limitation if a particular type of fiber is required. Secondly, the whole loop acts as the sensing element, being difficult to get accurate measurements from a specific location.

The all-PM FLM proposed in this work aims to combine the simplicity of the conventional one-section HiBi FLM with the reliability and robustness of all-PM setups. Three HiBi fiber sections are employed in the setup, two corresponding to the PM coupler (communication fibers) and the sensor itself. The contribution generated by the connecting fibers is canceled if both fibers (of the same type) present the same length and are spliced with a total angle offset between them of $90^{\circ}$. Since the influence of the communication fibers is cancelled, the interference pattern is defined only by the sensing fiber. The theoretical analysis of the setup must be done using the threesection analysis presented in [11]. For a three section FLM, the total transfer function of the interferometer is described by:

$$
T=\left[C_{1}+C_{2}+C_{3}+C_{4}\right]^{2}
$$

Where:

$$
\begin{aligned}
& C_{1}=\cos \left(\left(\beta_{1}+\beta_{2}+\beta_{3}\right) / 2\right) \sin \left(\theta_{c}\right) \cos \left(\theta_{2}\right) \cos \left(\theta_{3}\right) \\
& C_{2}=\cos \left(\left(-\beta_{1}+\beta_{2}+\beta_{3}\right) / 2\right) \cos \left(\theta_{c}\right) \sin \left(\theta_{2}\right) \cos \left(\theta_{3}\right) \\
& C_{3}=\cos \left(\left(\beta_{1}+\beta_{2}-\beta_{3}\right) / 2\right) \cos \left(\theta_{c}\right) \cos \left(\theta_{2}\right) \sin \left(\theta_{3}\right) \\
& C_{4}=-\cos \left(\left(\beta_{1}-\beta_{2}+\beta_{3}\right) / 2\right) \sin \left(\theta_{c}\right) \sin \left(\theta_{2}\right) \sin \left(\theta_{3}\right)
\end{aligned}
$$

Initially, it should be noted that the value $\theta_{c}=0^{\circ}$ is imposed by the PM optical coupler [13]. In addition, considering that fibers $l_{1}$ and $l_{3}$ are of the same type, their length must be equal $l_{1}=l_{3}$ to cancel their contributions: thus $\pm \beta_{1} \mp \beta_{3}=0$. Finally, the angles $\theta_{2}$ and $\theta_{3}$ are chosen so $\theta_{2}+\theta_{3}=90^{\circ}$ with the aim of maximizing the visibility of the interference. In order to avoid any influence induced by the communication fibers, the angles selected are $\theta_{2}=\theta_{3}=45^{\circ}$. Therefore, the final transfer function can be derived from (2), obtaining the equation described in (3). The result is equivalent to the behavior of the conventional FLM explained in (1) where the angle $\theta$ is set by the polarization controller and maximized to one $(\sin \theta=1)$.

$$
T=1-R=\left[2 \sin ^{2} 45 \cos \left(\beta_{2} / 2\right)\right]^{2}=\cos ^{2}\left(\beta_{2} / 2\right)
$$

The experimental setup used to verify the operation of the proposed system can be seen in Fig. 2. It consists of a PM coupler and a sensing fiber. The output ports of the coupler act as communication fibers and are made of Panda fiber SM15PS-U25A with a length of $l_{l}=l_{3}=21 \mathrm{~cm}$ and a beat-length of $3.98 \mathrm{~mm}$. These fibers are fused to the sensing fiber with an angle offset of $45^{\circ}$. The fiber section used as sensor is a Panda type HB1500 with a beat length of $3.8 \mathrm{~mm}$ and a total length of $38.2 \mathrm{~cm}$. The interrogation of the scheme is performed using a commercial FBG interrogator Smartec SM125 in combination with a custom software to perform the FFT.

\section{EXPERIMENTAL RESULTS}

\section{A. Validation of the all-PM fiber loop mirror}

First of all, it has been verified the correct operation of the proposed all-PM. In this respect, the optical transfer function of the scheme has been analyzed and compared with the simulated response employing (2) (with the parameters stated in the previous section). Additionally, $1 \mathrm{~dB}$ of power loss due to the fiber splices and coupler insertion loss have been considered. The measured and simulated transfer function of the all-PM interferometer can be seen in Fig. 3. The simulation agrees with the experimental results, with a little mismatch in the amplitude of the rejected band at 1538 and $1585 \mathrm{~nm}$. This is due to the difference between the lengths of the coupler arms in the setup. Ideally, the lengths were designed to be equal, but experimentally there is always a small mismatch. The length difference in this work is estimated to be $<1 \mathrm{~cm}$. Larger imbalances (several centimeters) would create new spatial frequency components as in [13].

To evaluate the performance of the scheme as sensor, it is employed a technique based on the FFT. As aforementioned, the measurements are carried out using a commercial FBG interrogator with a Matlab software that computes the FFT at a refresh rate of $1 \mathrm{~Hz}$ as in [11]. The optical response given by the interrogator is processed using the FFT obtaining magnitude and phase spectra. In this manner, a sinusoidal signal is identified by a peak in the magnitude spectrum located at a spatial frequency given by $S f=l /\left(L_{B} \lambda_{0}\right)$. The phase information of the sinusoid is represented in the FFT phase spectrum by the corresponding value at the spatial frequency $S f$. Fig. 4 shows the FFT spectra of the proposed scheme and its simulated response. It can be observed a good agreement in the location and amplitude of the interference peak, with a characteristic spatial frequency $S f=0.0625 \mathrm{~nm}^{-1}$.

Taking all this into consideration, the phase shifts in the optical spectrum induced by temperature/strain changes are also reflected in a variation in the value of the FFT phase at $0.0625 \mathrm{~nm}^{-1}$. Accordingly, this point of the FFT spectrum is monitored in the following section to get the temperature readings. It should be noted that FFT phase values are limited between $\pm \pi$. Nevertheless, there are not abrupt temperature changes during the refresh time (1s); thus, the phase data can be easily handled by software to extend the measurement range as needed. 


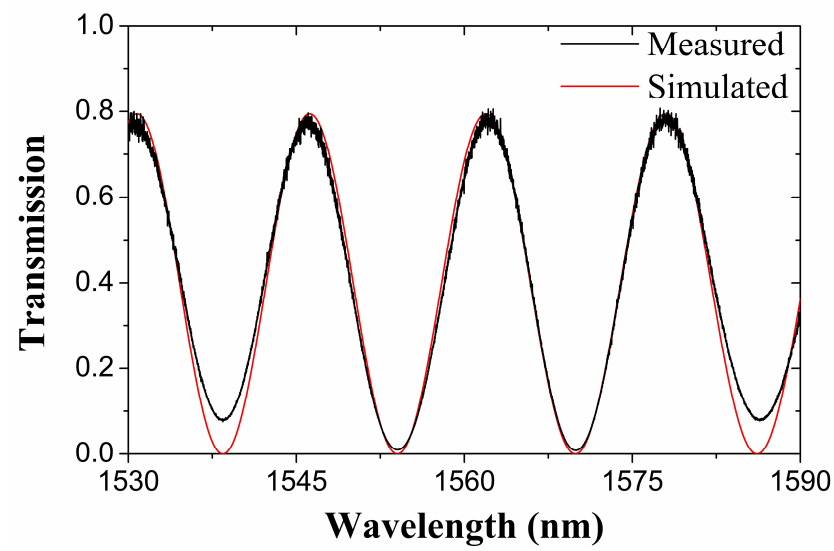

Fig. 3. Measured and simulated transfer function of the all-PM FLM.

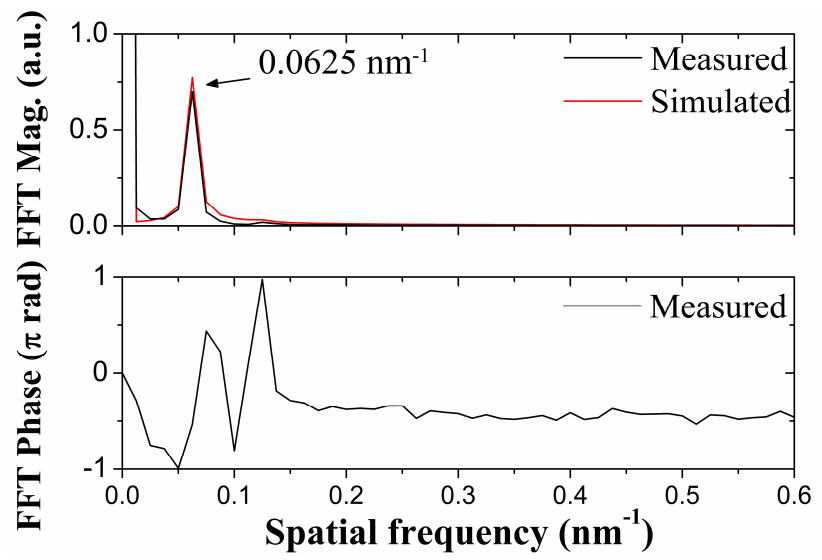

Fig. 4. Fast Fourier transform magnitude and phase spectra of the all-PM FLM transfer function.

\section{B. Simultaneous vibration and temperature sensing}

In the first experiment, the properties of the FFT-based interrogation technique are exploited to validate the scheme as sensor for mechanical vibration. In this case, it is analyzed the spatial frequency of a new peak generated in the magnitude FFT spectrum, instead of monitoring a value of the FFT phase (as in temperature measurements). The measurement principle is simple but relies on different properties which combined; allow the detection of mechanical vibration up to $1.5 \mathrm{kHz}$, using an interrogator with a refresh rate of $1 \mathrm{~Hz}$.

In the setup of the experiment, a mechanical wave driver applies transversal mechanical vibration to the sensing fiber which is fixed at both ends (Fig. 5). When the wave driver operates, the vibration generates a periodic axial-strain variation on the fiber. In this manner, there is a fast phase shift induced in the optical response of the interferometer at a frequency given by the frequency of the vibration (much higher than the refresh rate of the interrogator). However, the interrogator employs a tunable laser with a scan rate of $1 \mathrm{~Hz}$ in the $1510-1590 \mathrm{~nm}$ range. Thus, the optical response of the interrogator reflects the fast phase variation of the spectrum, which is translated to an amplitude modulation as seen in Fig. 6 for a vibration of $200 \mathrm{~Hz}$.

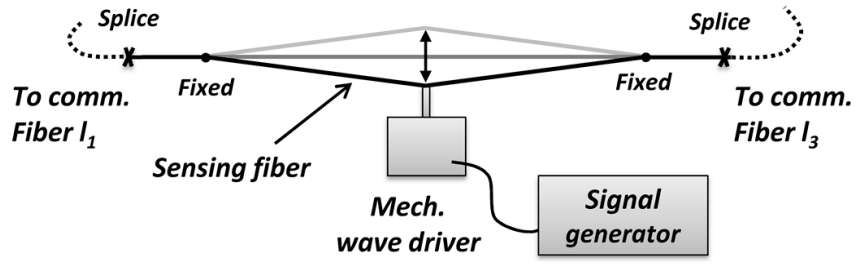

Fig. 5. Experimental setup for mechanical vibration measurements.

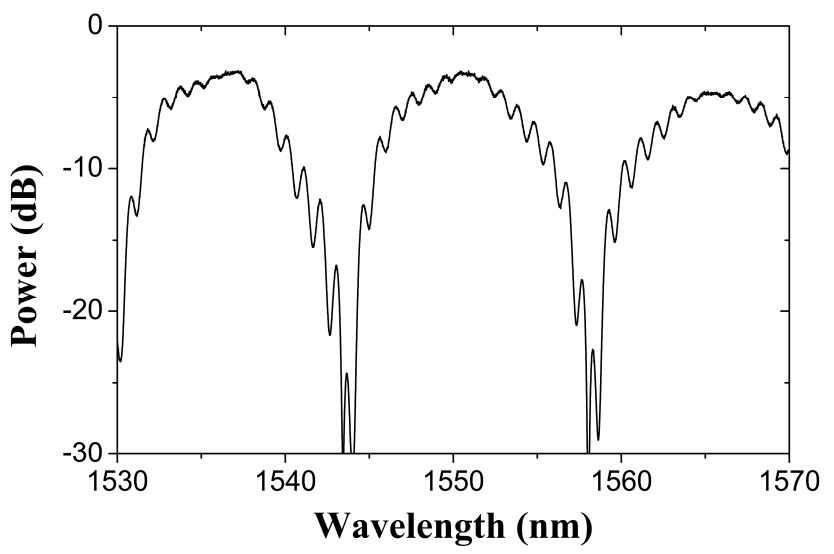

Fig. 6. Optical spectrum of the proposed all-PM FLM when a vibration of $200 \mathrm{~Hz}$ is applied.

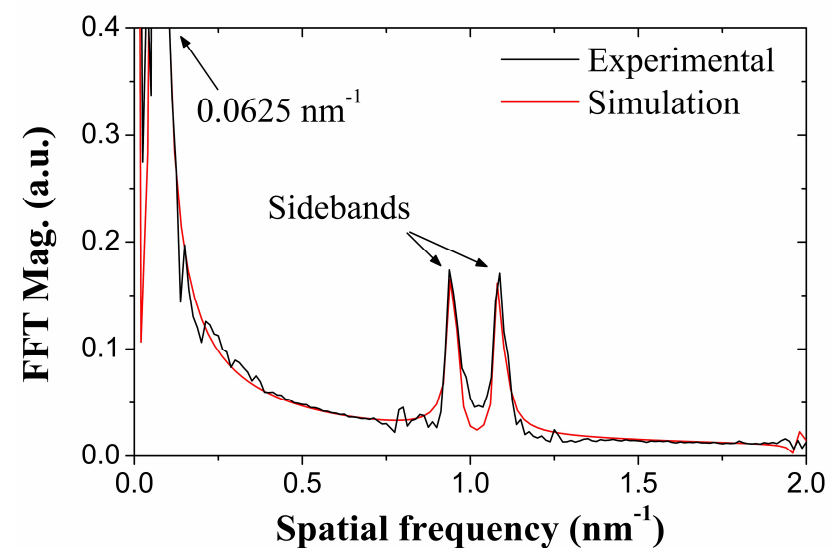

Fig. 7. Fast Fourier transform magnitude spectrum of the all-PM FLM when a vibration of $200 \mathrm{~Hz}$ is applied.

This effect can be understood as a narrowband phase modulation where the main FLM interference acts as the carrier, which is phase-modulated by the periodic mechanical vibration. Since the phase shift is small compared to the total period of the interference (otherwise the fiber would break due to an excessive strain), the modulation can be approximated to an amplitude modulation [18]. In accordance, the magnitude FFT spectrum presents a new pair of peaks at a spatial frequency given by the frequency of the mechanical vibration (Fig. 7). These two peaks are the sidebands of the induced amplitude modulation, each corresponding to the positive and negative frequency shift respectively. It should be clarified that only the spatial frequency of the sideband at highest spatial frequency is considered to quantify the frequency of the mechanical vibration. 


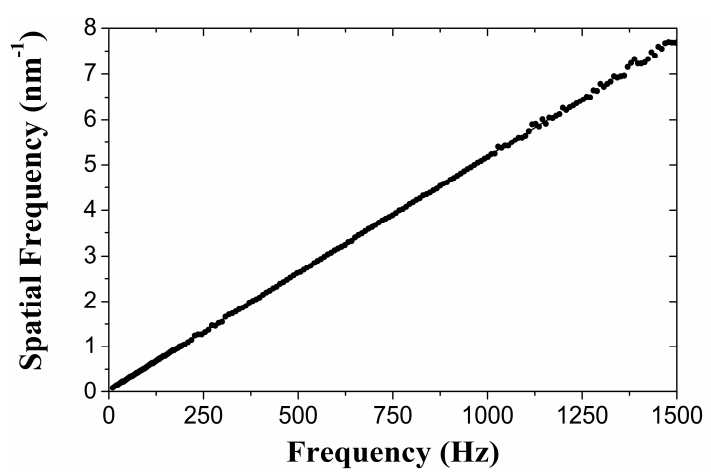

Fig. 8. Spatial frequency variation of the sideband against frequency of vibration.

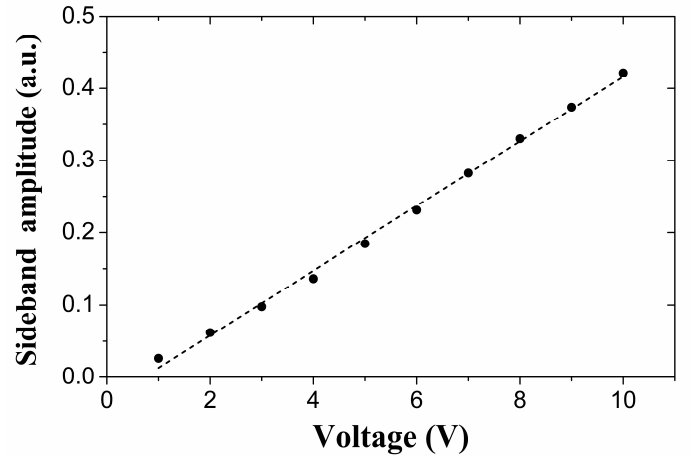

Fig. 9. Relationship between voltage applied to the mechanical wave driver and amplitude of the induced sideband.

In order to validate this interrogation technique, a frequency sweep was performed by the mechanical wave driver from 10 $\mathrm{Hz}$ to $1500 \mathrm{~Hz}$ using a sinusoidal signal with an amplitude that decreased with frequency due to mechanical limitations of the driver. Meanwhile, the spatial frequency of the generated sideband was monitored. As displayed in Fig. 8, the relationship between spatial frequency and vibration frequency is linear, with a sensitivity of $5.15 \mathrm{~nm}^{-1} / \mathrm{kHz}$ and a linear fitting factor $\mathrm{R}^{2}=0.9999$. The frequency resolution offered by this method is imposed the spatial frequency resolution of the FFT, which is $0.0125 \mathrm{~nm}^{-1}$; that corresponds to $\approx 2.4 \mathrm{~Hz}$. Regarding the frequency range that can be measured, the maximum limit in our experiment was imposed by the wave driver and its capability to generate mechanical vibration with amplitude enough to be detected at high frequencies. This small amplitude is the reason why the accuracy of the measurements decreases for high frequencies, as can be noticed in Fig. 8. On the other hand, the minimum frequency is limited by the relation between the vibration amplitude and the slope of the main contribution at $0.0625 \mathrm{~nm}^{1}$ (Fig. 7). It should be noted that these parameters are set by the wavelength range, optical resolution and refresh rate of the interrogator.

The amplitude of the induced modulation (and equivalently the amplitude of the generated sideband in the FFT spectrum) is directly proportional to the amplitude of the mechanical vibration applied to the sensor. To verify this, the amplitude of the sideband generated is depicted against the voltage set in the wave generator. The results can be seen in Fig. 9, confirming the linear relationship between the amplitude of the vibration and the amplitude of the sideband detected.

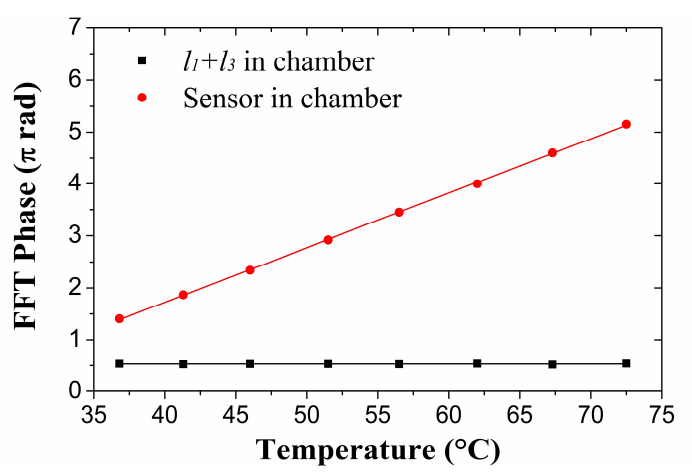

Fig. 10. Results of the temperature characterization (red dots) and crosstalk between communication and sensing fibers (black squares).

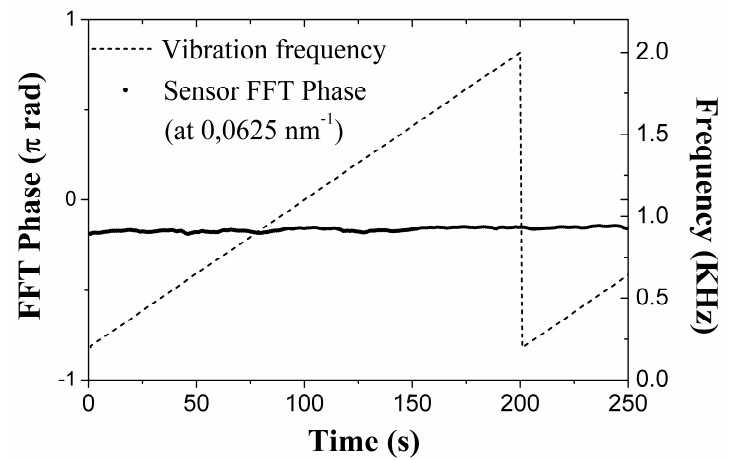

Fig. 11. FFT phase variation of the sensing fiber (at $0.0625 \mathrm{~nm}^{-1}$ ) as a function of the vibration applied.

After validating the system as a vibration sensor using the FFT, the all-PM FLM was tested as a temperature sensor by inserting the sensing fiber inside a climatic chamber. For this purpose, the FFT phase value located at $0.0625 \mathrm{~nm}^{-1}$ was monitored while a temperature sweep was performed from 35 to $75^{\circ} \mathrm{C}$. The results show a temperature sensitivity of $0.105 \pi$ $\mathrm{rad} /{ }^{\circ} \mathrm{C}$ with a linear fitting error $\mathrm{R}^{2}=0.9998$ (Fig. 10 red dots). With the aim of analyzing the crosstalk between the communication fibers $l_{1}, l_{3}$ and the sensing fiber, a second temperature sweep was carried out. In this case, only the fiber sections $l_{1}$ and $l_{3}$ were inserted in the chamber while the sensor remained at constant temperature. It can be observed in Fig. 10 (black squares) that there is no variation in the sensor response, validating the sensor operation without crosstalk.

Finally, it was evaluated the crosstalk between vibration and temperature measurements. In this respect, the FFT phase that includes the temperature information (located at $0.0625 \mathrm{~nm}^{-1}$ ) was recorded while a frequency sweep of the mechanical vibration was applied to the sensor. As depicted in Fig. 11, there is no crosstalk since the FFT phase of the sensing fiber at $0.0625 \mathrm{~nm}^{-1}$ (i.e. the temperature reading) remains constant during the variation of mechanical frequency. It should be mentioned that slight crosstalk from comm. fibers could appear if just one of the comm. fibers is subject to strain/temperature variations and $\theta_{2}$ or $\theta_{3} \neq 45^{\circ}$. Consequently, crosstalk can be suppressed to be negligible by two means: locating the comm. fibers close to each other and performing accurate splices. 


\section{Stability analysis versus conventional HiBi FLMs}

It has been mentioned in Section II that one of the main advantages of all-PM FLMs over conventional setups (that employ single-mode fiber) is their increased robustness and reliability. Single mode fibers are not purely symmetric, having certain degree of birefringence that depends on different aspects like temperature or bending. As a consequence, the polarization states inside the loop vary if the fiber suffers any disturbance. In practice, this is an important limitation because the whole interferometer must remain steady after the polarization controller has been adjusted. Even then, depending on environment changes, the system might require periodic adjustments of the polarization controller. In the case of an all-PM setup, the polarization states are preserved and cannot be adjusted. The polarization-preserving properties of the PM fibers are also influenced by external perturbations; but in general, the effect is not as severe as in the conventional setups.

In this regard, a series of measurements has been performed to analyze the reliability and robustness of the proposed allPM FLM and compare it to a conventional HiBi FLM. A conventional scheme was assembled by connecting the output ports of an optical coupler in a loop by means of a polarization controller and a section of $40 \mathrm{~cm}$ of Panda fiber (as explained in Section II A). In order to compare the behavior of the two sensors, the optical spectra were recorded while both interferometers were moved together, located and fixed in the climatic chamber for the temperature test; suffering approximately equal bending, torsion and strain changes. The process did not take place under controlled conditions; however, it is considered that it can describe well the different performances of conventional and all-PM fiber loop mirrors. During the test, some events can be easily identified due to the important effect on the conventional FLM. From 10 to 25 seconds, the fibers of the interferometers were untwisted, generating a strong phase and amplitude change in the conventional version, probably due to torsion changes. Around the $100 \mathrm{~s}$ the setups were placed and fixed in the climatic chamber (still off). Some alterations can be seen in the phase, with a deep amplitude drop that recovered soon due to a bend. Finally, between 175-200 s some strong instabilities are seen due to the pressure changes induced in the fiber by closing the door of the climatic chamber (which joint slightly pressed the comm. fibers).

The optical response of the conventional HiBi FLM during 200 s of this process is displayed in Fig. 12. It can be seen that the fringes visibility varied drastically during the experiment, even losing the interference for a certain period. Moreover, strong phase variations not related with strain or temperature fluctuations in the sensor are also detected. Conversely, the output of the all-PM setup remained considerably more stable (Fig. 13). There is some amplitude variation in the minima of the fringes that corresponds to external perturbations in the communication fibers $l_{1}$ and $l_{3}$. These perturbations are not perfectly compensated due to slight length difference between $l_{1}$ and $l_{3}$. In any case, the interference pattern remained almost constant and the phase is not modified.

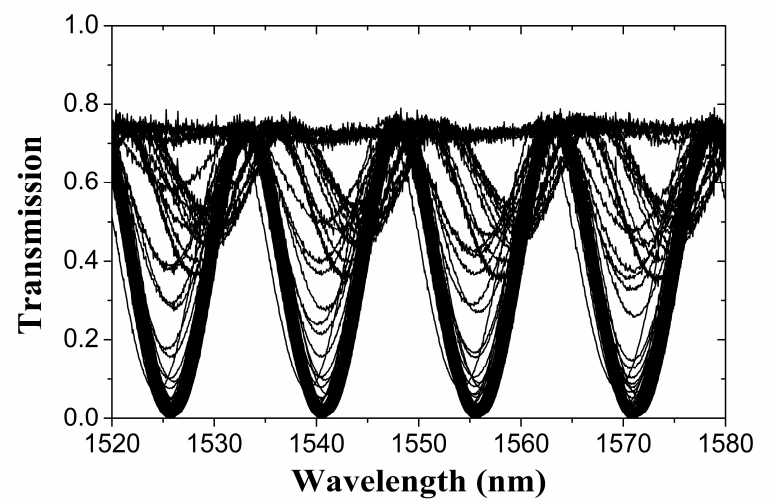

Fig. 12. Optical response of the conventional HiBi FLM during the test (200 traces).

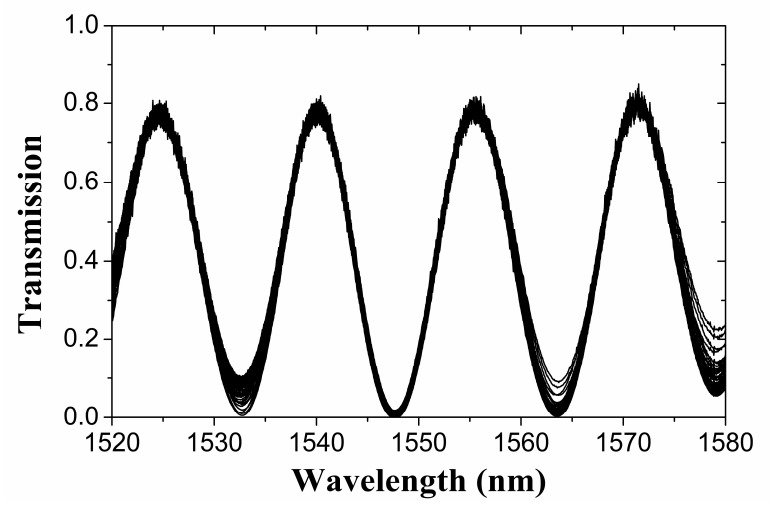

Fig. 13. Optical response of the proposed all-PM FLM during the test (200 traces).

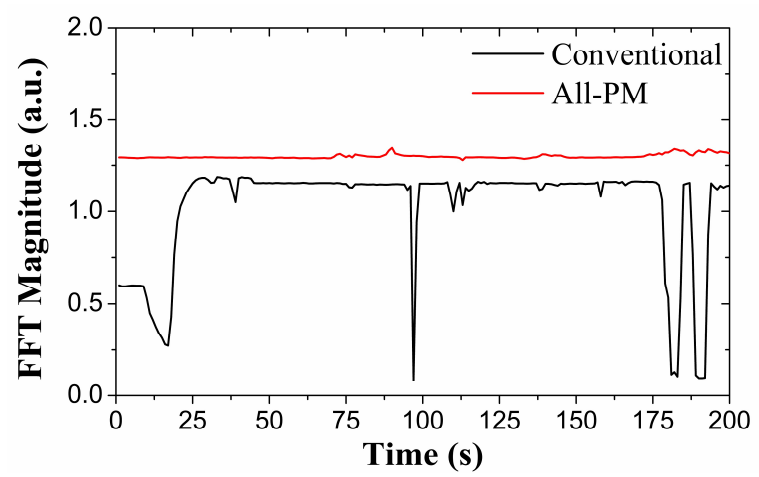

Fig. 14. Amplitude variation of the interference peak in the FFT magnitude spectrum during the test. Black: Conventional HiBi FLM. Red: Proposed all-PM FLM.

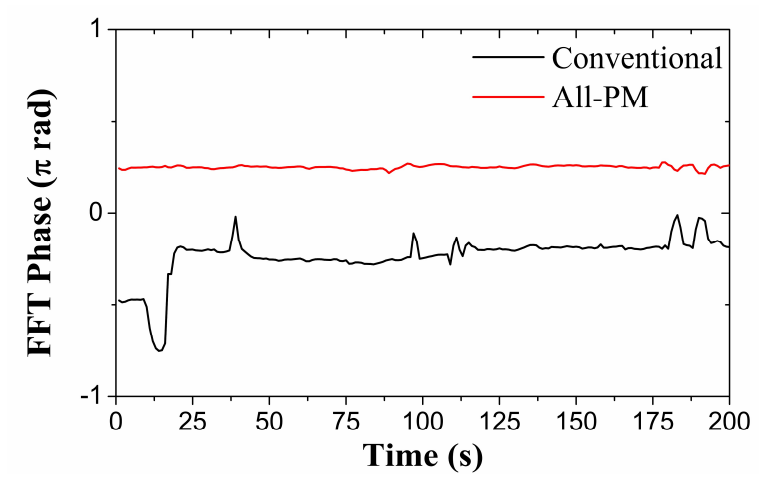

Fig. 15. FFT Phase variation at the spatial frequency of the sensors during the test. Black: Conventional HiBi FLM. Red: Proposed all-PM FLM. 
To show these results in a more comprehensive manner, the amplitude of the peak in the FFT and the phase variations of both sensors are represented during the process in Fig. 14 and 15. These results show the enhanced immunity of the all-PM setup to perturbations in the communication fibers. On the other hand, the conventional setup is strongly affected by perturbations in the single-mode fiber, drastically varying the visibility of the interference and more importantly the phase of the FFT, i.e. the sensor reading.

\section{CONCLUSION}

In this study, a new all-PM FLM interferometer has been proposed and analyzed. The system has been studied theoretically and compared with the conventional version that employs single-mode fiber. The design comprises a polarization-maintaining optical coupler which arms, of the same length, are fused to the sensing fiber with a rotation angle of $45^{\circ}$, with a total angle offset between them of $90^{\circ}$. In accordance, the interference contributions of the arms, which act just as communication fibers, are canceled, obtaining an interference pattern only dependent on the sensing fiber. As a consequence, the optical response of the interferometer combines the simplicity and well-defined sensing region of the simple one-section HiBi FLM with the advantages of all-PM setups. Simulations have been performed using the threesection theory, matching well with the experimental results.

The scheme has been validated as vibration and temperature sensor without crosstalk between measurands using an interrogation technique based on the fast Fourier transform. The mechanical vibration applied to the sensing fiber is detected by monitoring the sidebands generated in the magnitude spectrum of the FFT. In this manner, the amplitude and frequency of the mechanical vibration can be derived up to $1.5 \mathrm{kHz}$ employing a commercial FBG interrogator with a scan frequency of $1 \mathrm{~Hz}$ (i.e. without using fast detection/acquisition devices). On the other hand, the temperature readings can be obtained by analyzing the phase of the FFT at the spatial frequency that corresponds to the main contribution of the interference. The crosstalk-free operation has been verified between vibration and temperature but also between communication and sensing fibers. Finally, the behavior of the proposed scheme has been compared to the conventional under external perturbations in the communication fibers. The results show the high dependence of the conventional setup on the perturbations, decreasing and even losing the interference visibility. More importantly, a phase shift in the optical response unrelated with sensor variations was induced. Conversely, due to the proper compensation of the PM communication fibers, the all-PM setup remained virtually unaffected by external disturbances. This is an important property that can increase the practical applicability of $\mathrm{HiBi}$ fiber loop mirrors.

Summarizing, the proposed all-PM setup offer the benefits of all-PM setups (high resolution, accuracy, robustness against external perturbations and no need of polarization controllers) combined with a simple interference pattern and allowing the use of specific fibers as sensor. It is worth mentioning that the balanced-arms technique can be extended to multiplexing setups so one extra sensor can be monitored without increasing the number of frequency contributions. The main downside over conventional approaches is the need of a PM coupler, which increases the cost of the system. Moreover, splicing machines with rotation control are required to assemble the interferometer.

\section{REFERENCES}

[1] D. B. Mortimore, "Fiber loop reflectors," in Journal of Lightwave Technology, vol. 6, no. 7, pp. 1217-1224, Jul 1988.

[2] Y. Liu, B. Liu, X. Feng, W. Zhang, G. Zhou, S. Yuan, G. Kai and X. Dong,. "High-birefringence fiber loop mirrors and their applications as sensors," in Applied Optics, vol. 44, no. 12, pp. 2382-2390, Apr 2005.

[3] K. Naeem, B. H. Kim, B. Kim, and Y. Chung, "Simultaneous multiparameter measurement using Sagnac loop hybrid interferometer based on a highly birefringent photonic crystal fiber with two asymmetric cores," in Opt. Express, vol. 23, no. 3, pp. 3589-3601, 2015.

[4] R. M. Andre, M. B. Marques, P. Roy and O. Frazao, "Fiber Loop Mirror Using a Small Core Microstructured Fiber for Strain and Temperature Discrimination," in IEEE Photonics Technology Letters, vol. 22, no. 15, pp. 1120-1122, Aug 2010.

[5] Y. Hu and S. Chen, "Spatial frequency multiplexing of optical fiber sensor arrays," Opt. Lett. 20, 1207-1209 (1995)

[6] M. Nagatsuka, M. Koizumi, S. Tanaka, A. Wada and N. Takahashi, "Precise measurement technique of long period fiber grating sensors using Fourier transform method," 2017 25th Optical Fiber Sensors Conference (OFS), Jeju, 2017, pp. 1-4.

[7] D. D. A. Le, S. Lee and Y. G. Han, "Relative humidity sensor based on a few-mode microfiber knot resonator by mitigating group index difference," 2017 25th Optical Fiber Sensors Conference (OFS), Jeju, 2017, pp. 1-4.

[8] D. Barrera et al., "Low-Loss Photonic Crystal Fiber Interferometers for Sensor Networks," in Journal of Lightwave Technology, vol. 28, no. 24, pp. 3542-3547, Dec.15, 2010.

[9] Y. J. Rao, J. Jiang, C. X. Zhou, "Spatial-frequency multiplexed fiberoptic Fizeau strain sensor system with optical amplification," in Sensors and Actuators A: Physical, vol. 120, no. 2, pp. 354-359, 2005.

[10] L. Liu, Y. Gong, Y. Wu, T. Zhao, H. J. Wu, Y. J. Rao, "Spatial frequency multiplexing of fiber-optic interferometric refractive index sensors based on graded-index multimode fibers," in Sensors, vol. 12, no. 9 , pp. 12377-12385, 2012.

[11] D. Leandro, M. Bravo, A. Ortigosa and M. Lopez-Amo, "Real-Time FFT Analysis for Interferometric Sensors Multiplexing," in Journal of Lightwave Technology, vol. 33, no. 2, pp. 354-360, Jan.15, 2015.

[12] D. Leandro, A. Lopez-Aldaba, M. Bravo and M. Lopez-Amo, "Monitoring Multiple Hi-Bi Sensing Fibers in a Single Fiber Loop Mirror," in Journal of Lightwave Technology, vol. 34, no. 19, pp. 45434549, Oct.1, 2016.

[13] D. Leandro, M. Bravo, M. Lopez-Amo, "High resolution polarizationindependent high-birefringence fiber loop mirror sensor," in Optics Express, vol. 23, no. 24, pp. 30985-30990, 2015.

[14] M. Bravo, M. Fernandez-Vallejo, M. Echapare, M. López-Amo, J. Kobelke, K. Schuster, "Multiplexing of six micro-displacement suspended-core Sagnac interferometer sensors with a Raman-Erbium fiber laser," in Optics Express, vol. 21, no. 3, pp. 2971-2977, 2013.

[15] H.Y. Fu, et al., "Multiplexing of polarization-maintaining photonic crystal fiber based Sagnac interferometric sensors," in Optics Express, vol. 17, no. 21, pp. 18501-18512, 2009.

[16] A. N. Starodumov, L. A. Zenteno, D. Monzon, E. De La Rosa, "Fiber Sagnac interferometer temperature sensor," in Applied Physics Letters, vol. 70, no. 1, pp. 19-21, 1997.

[17] D. Leandro and M. Lopez-Amo, "Simultaneous temperature and vibration monitoring using an all-PM fiber loop mirror interferometer," 2017 25th Optical Fiber Sensors Conference (OFS), Jeju, 2017, pp. 1-4.

[18] S. Haykin, M. Moher, "Continuous-wave modulation," in Communication Systems, 5th ed. US: Wiley, 2000, ch. 2, sec. 2.7, pp. 111-113. 